\title{
Raise the Bar and Lower the Dose: Current and Future Strategies for Radiation Dose Reduction in Head and Neck Imaging
}

\author{
M. Ibrahim, H. Parmar, E. Christodoulou, and S. Mukherji
}

\begin{abstract}
SUMMARY: Technologic advances in CT have generated a dramatic increase in the number of CT studies, with a resultant increase in the radiation dose related to CT scanning. Such increase in radiation dose is becoming a concern for the radiology community, especially with increasing public awareness of the dose burden related to examinations. To cope with the increase in CT-related radiation exposure, it is becoming necessary to optimize CT imaging protocols and apply radiation dose reduction techniques to ensure the best imaging with the lowest radiation dose.
\end{abstract}

ABBREVIATIONS: ASIR = adaptive statistical iterative reconstruction algorithm; $\mathrm{ATCM}=$ automated tube current modulation; $\mathrm{CTDI}=\mathrm{CT}$ dose index; $\mathrm{DLP}=$ dose-length product; $\mathrm{FBP}=$ filtered back-projection

D espite the introduction of MR imaging, the overall frequency of CT studies in neuroimaging has failed to decrease. Indeed, the advent of helical and multidetector row CT with rapid acquisitions times and new diagnostic fields (eg, CT angiography, perfusion CT) has led to a further increase in CT examinations. As application of CT is becoming more widespread, there is an emergent need for radiation dose reduction to avoid a reversal of the risk-benefit ratio associated with this imaging technique. Individual patient doses are increasing with newer faster scanners because of volume scanning, thinner sections, overlapping scans, and increasing scan coverage. The as low as reasonably achievable principle (ALARA) emphasizes optimizing CT imaging protocols to achieve the lowest radiation dose possible while maintaining an optimal image quality.

Radiation dose is proportional to the amount of energy delivered by the photons within the $\mathrm{x}$-ray beam. This is generally dependent on the total number of the photons and the individual photon energy within the $\mathrm{x}$-ray beam, which is dictated by the image-acquisition parameters such as kilovolt(peak), milliampere, and x-ray tube rotation time, along with other factors such as section thickness, scan coverage, and pitch. Additionally, body habitus and size of the patient are important factors of the radiation dose delivered, which is especially relevant in pediatric patients and small adults. As a response to the increased concern of

From the University of Michigan Health System, Ann Arbor, Michigan.

Please address correspondence to Mohannad Ibrahim, MD, University of Michigan Health System, 1500 East Medical Center Dr, Ann Arbor, MI 48109; e-mail: mibrahim@med.umich.edu

- Indicates open access to non-subscribers at www.ajnr.org

http://dx.doi.org/10.3174/ajnr.A3473 the higher radiation dose, there have been several advances in dose-reduction techniques with the introduction of tube current modulation, peak voltage optimization, noise-reduction reconstruction algorithms, adaptive dose collimation, and improved detection-system efficiency. Such techniques not only address the increased concern about radiation dose but also are becoming a marketing tool. The radiology community, including radiologists, technicians, and physicists, should be familiar with such dose-reduction techniques and should optimize the imaging protocols to achieve the best images with a lower radiation dose.

\section{CT Radiation Dose Measurement}

CT is a unique imaging technique with continuous exposure around the patient as the gantry rotates to cover the region to be examined. Consequently, energy deposition is fairly uniform at fixed radial positions across the scanned plane with a relatively symmetric gradient from the surface toward the center of the patient because the patient is equally irradiated from all directions. CT is unlike plain radiography, in which exposure is highest at the skin entrance with continuous reduction of the dose toward the skin exit. Due to $\mathrm{x}$-ray scattering, deposition of the radiation beam energy will extend beyond the directly scanned volume into the adjacent tissues. Furthermore, the divergence of the beam and limited efficiency of the collimator contribute to energy deposition in tissues that are not imaged in the specific section. The radiation dose in the scanned section is the summation of the dose due to the direct beam in the scanned plane and dose contributions from radiation scattering from all the sections scanned before and after the specific section (Table 1).

The conventional metric representing the integrated dose of 
Table 1: Descriptions for MSAD and CTDI variants

\begin{tabular}{|c|c|}
\hline Dose Index & Description \\
\hline MSAD & $\begin{array}{l}\text { Average dose over } 1 \text { scan interval in the center } \\
\text { region of multiple scans }\end{array}$ \\
\hline CTDI & $\begin{array}{l}\text { Integrated dose profile for a single section, } \\
\text { normalized to the beam width; equivalent to } \\
\text { MSAD and measured by using multiple TLDs } \\
\text { or } 1 \text { ionization chamber }\end{array}$ \\
\hline $\mathrm{CTDI}_{100}$ & $\begin{array}{l}\text { Radiation dose index for } 100 \mathrm{~mm} \text { along the } \\
\text { length of an entire pencil ionization chamber }\end{array}$ \\
\hline $\mathrm{CTDI}_{\mathrm{w}}$ & $\begin{array}{l}\text { Weighted average of the CTDI } 100 \text { measured at } \\
\text { the periphery and at the center of phantoms }\end{array}$ \\
\hline $\mathrm{CTDI}_{\mathrm{vol}}$ & $\mathrm{CTDI}_{\mathrm{w}}$ divided by pitch \\
\hline DLP & $\mathrm{CTDI}_{\mathrm{vol}}$ multiplied by the length of the scan \\
\hline
\end{tabular}

Note:-TLD indicates thermoluminescent dosimeter; MSAD, multiple scan average dose.

the direct irradiation of the scanned volume and the scattered radiation from the adjacent scanned volumes is the CT dose index measured in milligrays ( $10^{-}$Gy). "CTDI" is defined as the dose profile of a single $\mathrm{x}$-ray tube rotation integrated over a scan length in the $\mathrm{z}$-direction and normalized to the table travel per tube rotation in a scan with a pitch of 1 . The CTDI is equivalent to the multiple scan average dose, which is the average dose in the center region of the scan range over which CT is performed. ${ }^{2}$ It can be measured either in air or in a phantom by using either a pencil ion chamber or a row of thermoluminescent dosimeters. A 16-cmdiameter phantom is used to represent a patient's head, and a $32-\mathrm{cm}$-diameter phantom is used to represent a patient's body. $\mathrm{CTDI}_{100}$, is a radiation dose index with integration-dose limits of $-50-50 \mathrm{~mm}$, where $100 \mathrm{~mm}$ is the length of the active volume of the pencil ionization chamber used for the measurement (integration of the dose profile). The use of this chamber assumes that its length is sufficient for a complete integration of the exposure due to the scatter radiation from a single tube rotation in the middle of the chamber length. The CTDI ${ }_{w}$ is the weighted average of the CTDI ${ }_{100}$ measured at the periphery and at the center of cylindric acrylic phantoms $\left(2 / 3 \times \mathrm{CTDI}_{100 \text {,periphery }}+1 / 3 \times\right.$ $\mathrm{CTDI}_{100 \text {,center }}$ ). The $\mathrm{CTDI}_{\mathrm{vol}}$ is defined as $\mathrm{CTDI}_{\mathrm{w}} /$ pitch. The value of CTDI ${ }_{\mathrm{vol}}$ multiplied by the length of the scan (in centimeters) is known as the dose-length product and is measured in units of milligray-centimeters. The DLP is commonly reported on the CT scanner for each CT study and is included in the patient dose report. When thinking about the potential dose due to the CT scan, the metric to take into consideration is the DLP because it contains both the CTDI, which is a measure of scanner output for a particular scanning technique, and the total scanning length along the patient's body. The CTDI descriptors are not accurate estimates of the radiation dose received by the patient. The CTDI descriptors represent the scanner radiation output when measured in a standardized phantom.

\section{CT Acquisition Parameters and Basics of Dose Reduction}

Recommendations for reducing radiation dose have mainly focused on limiting the radiation exposure. The US Food and Drug Administration has established guidelines to address the growing concern over CT-associated radiation dose. ${ }^{3}$ These guidelines give recommendations on how to optimize CT protocols and encourage the elimination of inappropriate referrals for CT with reduction of the number of unnecessary repeat examinations. The basic pillars of dose reduction include justification of the study and eliminating inappropriate $\mathrm{CT}$ referrals, limiting scan range to the region of interest, limiting the number of contrast phases, and use of a relatively large pitch. The goal is an ALARA radiation dose.

There are several acquisition parameters and factors that have a direct effect on radiation dose, such as the photon energy (determined by the tube potential [kilovolt (peak)]); photon fluence, determined by the tube milliamperes; rotation or exposure time; reconstructed section thickness; object thickness or attenuation; pitch and/or spacing of the consecutive sections; and distance from the $\mathrm{x}$-ray tube to the isocenter. ${ }^{4}$ The basic strategies in minimizing radiation dose involve changes in the acquisition parameters (kilovolt(peak), milliampere, $\mathrm{x}$-ray tube rotation time, pitch) to achieve a lower radiation dose with an acceptable quantum mottle. Typically, if one of these parameters is decreased, another needs to be increased to maintain image quality. The effect of CT acquisition parameters on image quality and noise is interlinked and complex. The noise in a CT image is determined by several factors, with the number of the photons reaching the detectors (quantum mottle) having the most dominant role.

Beam Energy. The energy of the incident $\mathrm{x}$-ray beam is determined by the tube voltage or kilovolt(peak). Any variation in tube potential causes substantial change in the CT radiation dose. When all other parameters are held constant and the kilovolt(peak) is decreased, the radiation dose will also decrease. The relationship between the change in effective dose and tube potential is exponential. ${ }^{5}$

The effect of peak voltage on image quality is complex because it affects both image noise and tissue contrast. Image contrast is affected by the mean photon energy of the x-ray beam and the imaged material. ${ }^{6}$ In general, lower kilovolt(peak) yields better contrast, especially for bone and iodine, with the effect being much smaller for soft tissues like fat and muscle. ${ }^{6}$ The effect of improved image contrast at the lower kilovolt(peak) (while maintaining a constant milliampere-second) is reduced by the increased noise, especially with thick objects and near-dense bone, where the beam-hardening effect can, in fact, result in loss of the improved contrast achieved with a lower kilovolt(peak). The increase in image noise when lowering the kilovolt(peak) is most significant in individuals with larger body habitus, with the benefit of lowering the kilovolt(peak) best seen in pediatric patients and small adults. Lowering the kilovolt(peak) settings and increasing the milliampere-second, either by using automatic tube current modulation or by using a technique chart, is considered an effective strategy in lowering radiation dose while maintaining image quality. ${ }^{7-9}$

Moreover, lowering the kilovolt(peak) increases vascular enhancement because the attenuation of iodine-based contrast agents increases with reduced photon energy distribution due to the high atomic number of iodine and the effect of the iodine $\mathrm{k}$-edge in the x-ray attenuation at such energy levels. By reducing the kilovolt(peak), the mean photon energy in the $\mathrm{x}$-ray beam approaches the energy of the k-edge of the iodinated contrast media, which increases the $\mathrm{x}$-ray attenuation coefficient and yields an improved contrast enhancement without a decrease in image quality. ${ }^{10}$ This will particularly improve the conspicuity of hypervascular pathologies (Fig 1). 


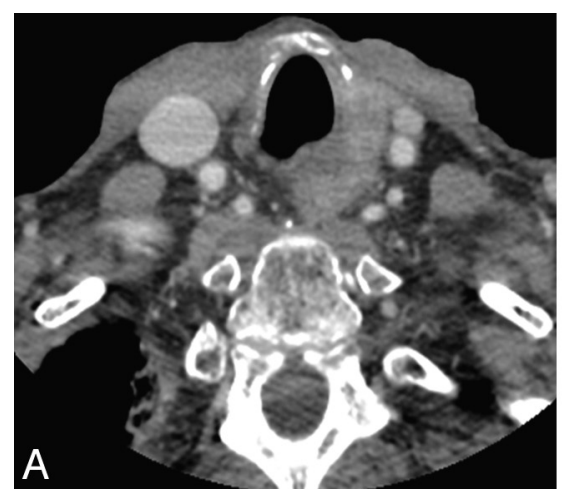

FIG 1. Patient scanned with a protocol at $140 \mathrm{kV}(\mathrm{p})$ (CTDI ${ }_{\mathrm{vol}}, 14.26$; W450, L75) (A) and $100 \mathrm{kV}(\mathrm{p})$ $\left(C\right.$ CTI $_{\text {vol }}, 8.26$; W450, L75) (B). Note the improved contrast and increased attenuation of the vessels (carotid arteries and jugular veins) and vertebral bodies and increased contrast of the metastatic node in the left tracheoesophageal groove with the lower kilovolt(peak) technique.

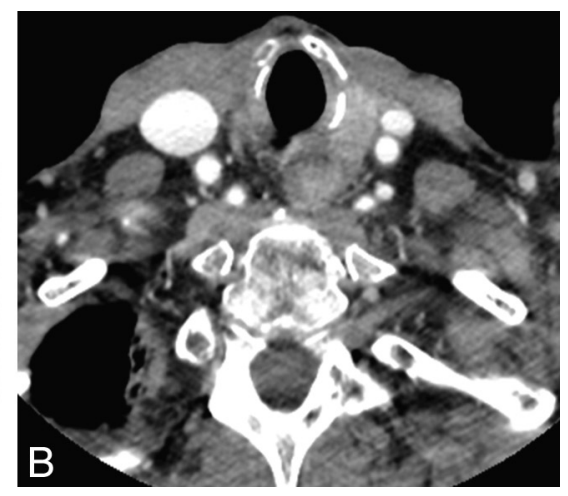

pitch). ${ }^{14}$ In such scanners, a constant effective milliampere-second value is held, irrespective of pitch value, by adjusting the tube current and increasing the tube current-time product approximately proportional to the increased pitch (or decreasing the tube current-time product proportional to the decreased pitch). This will keep the radiation dose relatively constant despite a variation of pitch. Additionally, an increase in the pitch value in modern scanners equipped with automatic milliampere modulation (see below) will not necessarily lead to dose reduction because the milliampere will be automatically adjusted to account for the increase in pitch.

Photon Fluence. The photon fluence, determined by the tube current (milliampere) and x-ray tube rotation time (second), has a direct effect on patient radiation dose. The radiation dose is directly proportional to the milliampere-second value; with the radiation dose increasing linearly with increasing milliamperesecond. Generally, decreasing (or increasing) the tube current by $50 \%$ will decrease (or increase) the radiation dose by half. Any decrease in tube current should be considered carefully because such reduction causes an increase in image noise, which may affect the diagnostic outcome of the examination. ${ }^{11}$ Image noise in CT is dominated by quantum mottle, which is determined by the number of photons incident, and is collected by the detector. The number of the photons is proportional to the milliampere-second. The electronic noise also affects the image noise and is the result of fluctuation of the electronic components of the dataacquisition system. Increasing the milliampere-second will increase the number of photons emitted from the $\mathrm{x}$-ray tube but will not change the energy spectrum of the photons.

Collimation, Table Speed, and Pitch. The helical or spiral acquisition involves continuous gantry rotation while simultaneously translating the table through the gantry during data acquisition. New acquisition parameters are being introduced in helical imaging such as table speed and pitch, which is defined as the ratio of table feed per gantry rotation to the nominal beam width. The pitch value has a direct influence and is inversely proportional to the radiation dose of the patient (Dose $\sim 1 /$ Pitch). ${ }^{12,13}$ This is because any increase in the pitch decreases the duration of the exposure to any particular section of the patient per gantry rotation and increases the gaps between successive gantry rotations.

Beam collimation, table speed, and pitch are interlinked parameters that affect the diagnostic quality and radiation dose of an imaging study. Faster table speed for a given collimation, resulting in higher pitch, is associated with a lower radiation dose because of a shorter exposure time. Although smaller beam collimation will result in a higher degree of overlap between the adjacent scans, this yields only minimal change in the radiation dose in comparison with a larger collimation for a given collimation and table speed. ${ }^{12}$ The effect of pitch on radiation dose and image quality is negated in scanners that use an "effective milliamperesecond setting" (defined as milliampere-second divided by
Although scanning at a higher pitch value is dose-efficient, a higher pitch value causes helical artifacts and degradation of the section sensitivity profile with increased volume averaging and a potential decrease in spatial resolution. Furthermore, noise is dependent on pitch in multidetector row CT because the spiral interpolation algorithm will make use of the redundant data acquired by different detector rows and will decrease the noise for pitch values of $<1$ (and increase it for a pitch value of $>1$ ). ${ }^{12}$

\section{Current Strategies in Dose Reduction}

Automated Tube Current Modulation. The most widely available technical innovation for significant radiation dose reduction is automated tube current modulation, also known as automatic exposure control. This technique allows constant image quality in the CT examination at a lower radiation dose, regardless of the patient size or the attenuation characteristics of the body part being scanned. Tube current modulation may be preprogrammed by determining the attenuation values obtained by refined analysis of the projection scouts obtained at the start of the examination (Fig $2 A$ ) or may be adjusted by using a feedback circuit with near real-time adjustment of the tube current based on the attenuation values of the preceding image or may incorporate a combination of preprogramming and feedback circuit.

ATCM enables automatic adjustment of the tube current in the xy plane (angular modulation), along the z-axis (longitudinal modulation), or both (combined modulation) to maintain a userselected noise level in the image (Fig $2 B,-C) .{ }^{15}$ Longitudinal modulation adjusts the tube current along the $\mathrm{z}$-axis, which results in a lower tube current in the neck region with a higher tube current at the skull base and thoracic inlet, rendering images of similar noise, independent of patient size or anatomy. In angular modulation, the tube current is adjusted in each angular projection within the same section, with increased tube current in the lateral projection compared with the anteroposterior projection. Depending on the manufacturer, ATCM systems operate on the basis of several methods: noise index (GE Healthcare, Milwaukee, Wisconsin), standard deviation (Toshiba Medical Systems, Tokyo, Japan), reference image (Philips Healthcare, Best, the Netherlands), and quality reference milliampere-second (Siemens, Erlangen, Germany) (Table 2). ${ }^{15}$ There have been several studies 

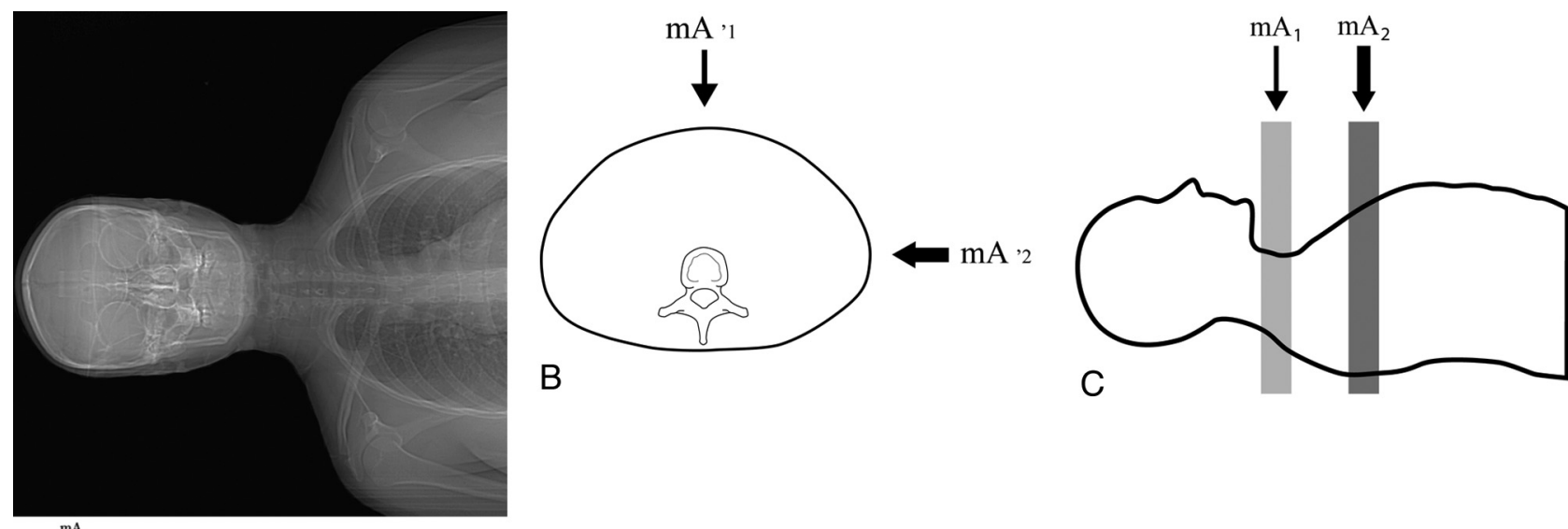

A

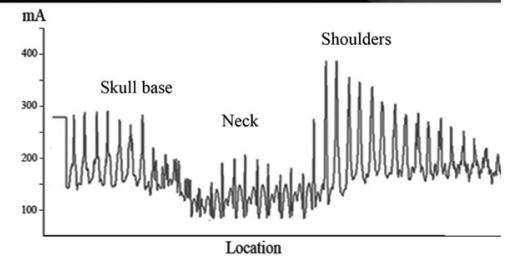

FIG 2. Schematic illustration demonstrating the modulation of the tube current for neck CT with variable milliampere values in each location based on the automatic analysis of attenuation values from the scout image $(A)$. Schematic illustration of the angular tube current modulation at 2 different angles in the xy plane $(B)$ and modulation of the milliampere along the $z$-axis $(C)$.

Table 2: Automated tube current modulation: manufacturer and principle

\begin{tabular}{|c|c|c|c|}
\hline Manufacturer & Trade Name & $\begin{array}{c}\text { Image } \\
\text { Quality Reference }\end{array}$ & Principle \\
\hline GE Healthcare & AutomA, SmartmA & Noise index & $\begin{array}{l}\text { Maintain a constant noise level (defined as noise index), using } \\
\text { tube current within prescribed minimum and maximum values }\end{array}$ \\
\hline Toshiba & Sure Exposure & SD & $\begin{array}{l}\text { Maintain a constant noise level (defined in standard deviation), } \\
\text { using tube currents and preset minimum and maximum values }\end{array}$ \\
\hline Siemens & CARE Dose4D & $\begin{array}{l}\text { Quality reference } \\
\text { milliampere-second }\end{array}$ & $\begin{array}{l}\text { Maintain the same image quality with reference to target effective } \\
\text { milliampere-second levels for standard-size patients }\end{array}$ \\
\hline Philips & DoseRight & Reference image & Keep the same image quality as in the reference image \\
\hline
\end{tabular}

that showed variable and sometimes significant levels of dose reduction with use of ATCM, depending on body region and patient size. ${ }^{16-18}$ It is important to be familiar with the different parameters of the ATCM technique to achieve appropriate image quality for each specific diagnostic task, to not generate images with lower noise than necessary. An appropriate minimum and maximum milliampere value with a designated noise index or SD (depending on the manufacturer) based on the observer preference is prudent to prevent an inappropriate increase in the image noise related to lower photon fluence.

Adaptive Dose Shielding. Helical CT imaging acquires additional data with a number of extra gantry rotations before the beginning and after the end of the scanned volume, a process called "overranging" (or " $z$ overscanning"). The extra data are needed for the interpolation required in the image reconstruction of a helical acquisition of the scanned volume. Consequently, tissue that will never be part of the reconstructed images will be exposed to radiation. This factor is especially significant with the trend toward developing scanners with greater detector collimation because the dose increase from over-ranging is proportional to pitch and collimation. ${ }^{19}$ To reduce the impact of over-ranging, it is better to use an axial protocol rather than a helical protocol, or by reducing the pitch or using narrower detector collimation. The adaptive dose shield is a technology based on precise and independent movement of collimator blades that limits this over-ranging (Fig 3 ). The collimator will asymmetrically open at the beginning and close at the end of each spiral scan, blocking the parts of the x-ray beam that are not used for image reconstruction. The proportion of radiation dose reduction depends on the scan range, detector collimation, and pitch and has been shown to range from $7 \%$ to $38 \% .^{19,20}$

Image Reconstruction Algorithms. CT image reconstruction from raw data was first performed by using an iterative reconstruction algorithm, which was computationally demanding and resulted in a relatively long reconstruction time. Iterative reconstruction accurately models the data-collection process in CT by generating a set of synthesized projections. The model incorporates details of the geometric information of the scanner (including dimensions of the focal spot, the size of each detector cell, and the shape and size of each image voxel) and system statistical information (including photon statistics and electronic noise in the data-acquisition system). ${ }^{21}$ Iterative reconstruction starts with an initial estimate of the object, which is iteratively improved in a stepwise fashion by comparing the synthesized projection with the acquired projection data and making an incremental change to the previous guess. ${ }^{21}$ Iterative reconstruction was 


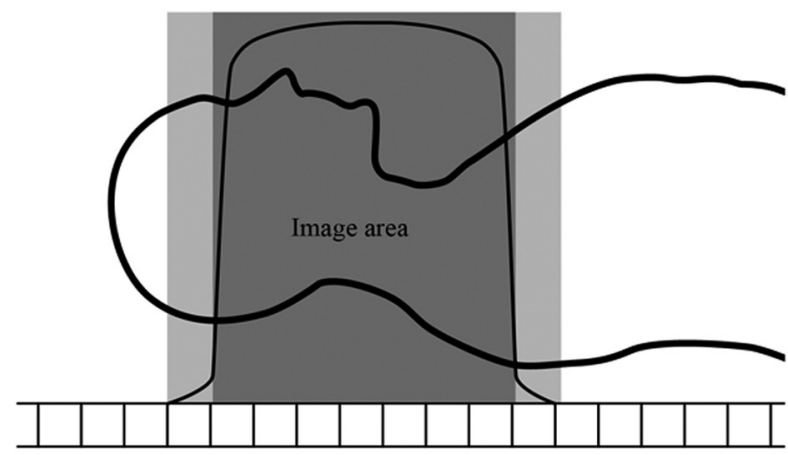

A

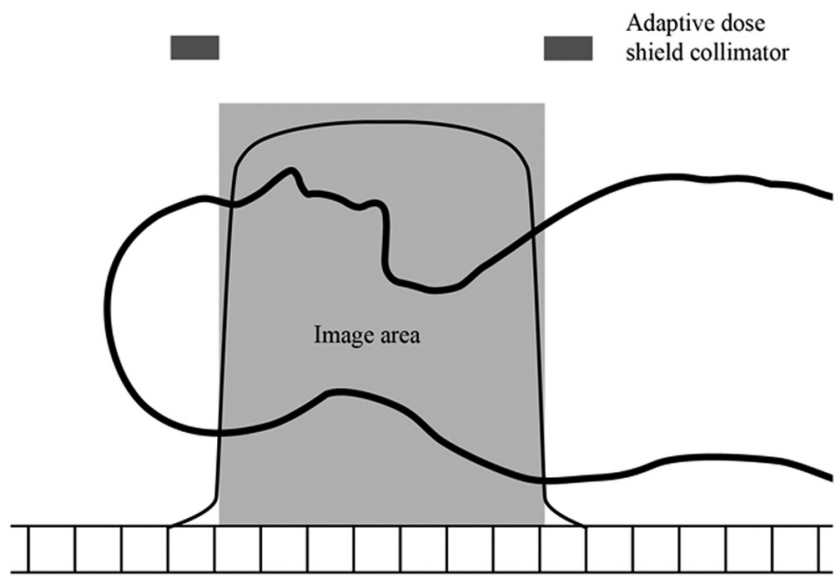

B

FIG 3. Schematic illustration of over-ranging $(A)$ and the effect of the adaptive dose shield technique $(B)$. The area marked in light gray in $A$ represents the range along the $z$-axis that is exposed by the extra gantry rotation, before and after the imaged volume, which is needed for image reconstruction during a helical scan. With adaptive dose shield $(B)$, a collimator closes asymmetrically at the beginning and the end of the examination, while opening fully in the center of the scan range.

quickly replaced by a filtered back-projection technique, which is an analytic reconstruction technique that operates on several fundamental assumptions about the scanner geometry and offers a compromise between reconstruction speed and image noise. A major drawback for the FBP algorithm is increased image noise that stems from the fact that FBP assumes noiseless projection data, which then must be overpowered by increasing the radiation dose. Iterative reconstruction reduces image noise without compromising spatial resolution, while in FBP, higher spatial resolution is achieved with higher image noise. With the increasing computational power in the recent decade, iterative reconstruction has come to focus on noise suppression and artifacts reduction associated with lowering the radiation dose. Major CT manufacturers are implementing their own iterative image-reconstruction methods to achieve dose reduction without imagequality degradation. These include the adaptive statistical iterative reconstruction algorithm and, more recently, a model-based iterative reconstruction technique (VEO) from GE Healthcare; Iterative Reconstruction in Image Space, and Sinogram-Affirmed Iterative Reconstruction from Siemens; Adaptive Iterative Dose Reduction from Toshiba ${ }^{22}$; and iDose from Philips Healthcare.

ASIR (GE Healthcare) is a modified iterative-reconstruction technique that starts iterative reconstruction after a first-pass FBP reconstruction. ${ }^{23}$ It models the photon statistics in $\mathrm{X}$-ray attenuation but does not model the system geometrics. Thus, it is more computationally complex than FBP but considerably less computationally complex than more comprehensive iterative reconstruction methods. ASIR can help shorten the longer reconstruction time of iterative reconstruction while maintaining much lower image noise when constructing the same raw data by using the FBP algorithm, allowing radiation dose reduction with no change in spatial or temporal resolution of the CT image. ${ }^{24}$ ASIR images may appear to have an unusual "texture," particularly when the radiation dose used is not sufficiently low. In clinical practice, using variable blending levels of image reconstruction with FBP and ASIR techniques can be performed to generate clinically acceptable images.

\section{Future Advancement in Dose Reduction}

Automated Organ-Based Current Modulation. This technique reduces the tube current for certain projections to avoid direct exposure of radiosensitive organs, for example the thyroid gland and ocular lens. ${ }^{25}$ It modulates the current along the $\mathrm{z}$-axis according to the body habitus and along the $\mathrm{x}$ and $\mathrm{y}$ planes by reducing the tube current over a $120^{\circ}$ radial arc prescribed over the anterior, lateral, or posterior aspect of the body (based on the operator preference). The overall radiation dose in the cross-sectional plane will remain constant, while decreasing the dose over the $120^{\circ}$ radial arc and increasing the dose in the remaining $240^{\circ}$ arc. Lowering the dose in the anterior projection by using organbased current modulation will limit direct exposure to the thyroid gland and the ocular lens while preserving image quality. ${ }^{25}$ The dose in the prescribed projection can be near zero, which will take advantage of the fact that only $180^{\circ}$ of data and the fan angle are necessary to reconstruct a CT image. ${ }^{26}$ The radio-sensitive organs, for example the thyroid gland, will be indirectly exposed to the beam coming from the posterior projection; however, this beam has been attenuated and filtered by the soft tissues of the patient.

Automated (Optimized) Tube-Voltage Modulation. Conventional dose-modulation techniques will modulate the tube current, while the tube voltage, the kilovolt(peak), setting is left unchanged. However, there is a large potential for dose reduction by optimizing the tube kilovolt(peak) setting. Such optimization of the kilovolt(peak) setting can be performed automatically for each individual patient and specific examination, by analyzing the information gathered by the topogram (scout view) to optimize kilovolt(peak) and milliampere-second to maintain a certain contrast-to-noise ratio. This will improve the selection of kilovolt(peak) for a particular examination beyond manual kilovolt(peak) selection.

Noise-Reduction Algorithm with Image Reconstruction and Data Processing. Improving the overall image quality with lower noise levels can be achieved by using optimally designed data processing 
and image reconstruction methods without sacrificing other image properties. Several noise-control techniques have been developed, operating on the raw projection data, the log-transformed sinogram, or the images after reconstruction, to achieve a lower noise in CT images. Computational advancement is allowing sophisticated reconstruction methods to be used to control noise and streak artifacts in the projection data domain before the image reconstruction. Because CT manufacturers are presenting different iterative image reconstruction methods to achieve lower noise, newer versions of such methods are being introduced, allowing further reduction of CT image noise and hence radiation dose. For example, a model-based iterative reconstruction algorithm (VEO; GE Healthcare) has been introduced following ASIR to achieve further noise reduction and a lower radiation dose. ${ }^{27}$ Additionally, several image-based filtering techniques usually perform quite well with regard to reducing image noise while maintaining high contrast resolution. ${ }^{28,29}$

\section{CONCLUSIONS}

There are significant variations between sites and scanners in imaging protocols with a wide range of radiation doses for the same scan indication. Furthermore, adult scan protocols sometimes have been directly applied to pediatrics without making proper adjustments. Consequently, there is true demand to optimize protocols and to become familiar with the factors affecting the CT radiation dose and available dose-reduction options. Several dose-reduction techniques have been successfully implemented and have been shown to reduce radiation exposure, including tube-current modulation, reducing tube voltage, adaptive dose shielding, and noise reduction filters. The increased noise and degraded image quality related to using a lower radiation dose have been successfully improved by using advanced image reconstruction techniques.

Disclosures: Suresh Mukherji—UNRELATED: Consultancy: Philips Medical Systems.

\section{REFERENCES}

1. Shope TB, Gagne RM, Johnson GC. A method for describing the doses delivered by transmission $x$-ray computed tomography. Med Phys 1981;8:488-95

2. Bauhs JA, Vrieze TJ, Primak AN, et al. CT dosimetry: comparison of measurement techniques and devices. Radiographics 2008;28:245-53

3. Feigal DW Jr. FDA public health notification: reducing radiation risk from computed tomography for pediatric and small adult patients. Int J Trauma Nurs 2002;8:1-2

4. McNitt-Gray MF. AAPM/RSNA physics tutorial for residents: topics in CT-radiation dose in CT. Radiographics 2002;22:1541-53

5. Schindera ST, Nelson RC, Yoshizumi T, et al., Effect of automatic tube current modulation on radiation dose and image quality for low tube voltage multidetector row CT angiography: phantom study. Acad Radiol 2009;16:997-1002

6. Huda W, Scalzetti EM, Levin G. Technique factors and image quality as functions of patient weight at abdominal CT. Radiology 2000;217: $430-35$

7. Mulkens TH, Marchal P, Daineffe S, et al. Comparison of low-dose with standard-dose multidetector CT in cervical spine trauma. AJNR Am J Neuroradiol 2007;28:1444-50

8. Sigal-Cinqualbre AB, Hennequin R, Abada HT, et al., Low-kilovoltage multi-detector row chest $\mathrm{CT}$ in adults: feasibility and effect on image quality and iodine dose. Radiology 2004;231:169-74
9. Waaijer A, Prokop M, Velthuis BK, et al. Circle of Willis at CT angiography: dose reduction and image quality - reducing tube voltage and increasing tube current settings. Radiology 2007;242:832-39

10. Matsuoka S, Hunsaker AR, Gill RR, et al. Vascular enhancement and image quality of MDCT pulmonary angiography in 400 cases: comparison of standard and low kilovoltage settings. AJR Am J Roentgenol 2009;192:1651-56

11. Kalra MK, Maher MM, Toth TL, et al. Strategies for CT radiation dose optimization. Radiology 2004;230:619-28

12. Primak AN, McCollough $\mathrm{CH}$, Bruesewitz MR, et al. Relationship between noise, dose, and pitch in cardiac multi-detector row CT. Radiographics 2006;26:1785-94

13. McNitt-Gray MF, Cagnon $\mathrm{CH}$, Solberg TD, et al. Radiation dose in spiral CT: the relative effects of collimation and pitch. Med Phys 1999;26:409-14

14. Mahesh M, Scatarige JC, Cooper J, et al. Dose and pitch relationship for a particular multislice CT scanner. AJR Am J Roentgenol 2001; 177:1273-75

15. Lee $\mathrm{CH}$, Goo JM, Ye HJ, et al. Radiation dose modulation techniques in the multidetector CT era: from basics to practice. Radiographics 2008;28:1451-59

16. Russell MT, Fink JR, Rebeles F, et al. Balancing radiation dose and image quality: clinical applications of neck volume CT. AJNR Am J Neuroradiol 2008;29:727-31

17. Lee EJ, Lee SK, Agid R, et al. Comparison of image quality and radiation dose between fixed tube current and combined automatic tube current modulation in craniocervical CT angiography. AJNR Am J Neuroradiol 2009;30:1754-59

18. Mulkens TH, Bellinck P, Baeyaert M, et al. Use of an automatic exposure control mechanism for dose optimization in multi-detector row CT examinations: clinical evaluation. Radiology 2005;237:213-23

19. Tzedakis A, Damilakis J, Perisinakis K, et al. The effect of $\mathbf{z}$ overscanning on patient effective dose from multidetector helical computed tomography examinations. Med Phys 2005;32:1621-29

20. Christner JA, Zavaletta VA, Eusemann CD, et al. Dose reduction in helical CT: dynamically adjustable $\mathrm{z}$-axis $\mathrm{x}$-ray beam collimation. AJR Am J Roentgenol 2010;194:W49-55

21. $\mathrm{Xu} \mathrm{J}$, Mahesh $\mathrm{M}$, Tsui $\mathrm{BM}$. Is iterative reconstruction ready for MDCT? J Am Coll Radiol 2009;6:274-76

22. Yoo RE, Park EA, Lee W, et al. Image quality of Adaptive Iterative Dose Reduction 3D of coronary CT angiography of 640-slice CT: comparison with filtered back-projection. Int J Cardiovasc Imaging 2012 Aug 26. [Epub ahead of print]

23. Silva AC, Lawder HJ, Hara A, et al. Innovations in CT dose reduction strategy: application of the adaptive statistical iterative reconstruction algorithm. AJR Am J Roentgenol 2010;194:191-99

24. Kilic K, Erbas G, Guryildirim M, et al. Lowering the dose in head CT using adaptive statistical iterative reconstruction. AJNR Am J Neuroradiol 2011;32:1578-82

25. Hoang JK, Yoshizumi TT, Choudhury KR, et al. Organ-based dose current modulation and thyroid shields: techniques of radiation dose reduction for neck CT. AJR Am J Roentgenol 2012;198;1132-38

26. Vollmar SV, Kalender WA. Reduction of dose to the female breast in thoracic CT: a comparison of standard-protocol, bismuthshielded, partial and tube-current-modulated CT examinations. Eur Radiol 2008;18:1674-82

27. Katsura M, Matsuda I, Akahane M, et al. Model-based iterative reconstruction technique for radiation dose reduction in chest $\mathrm{CT}$ : comparison with the adaptive statistical iterative reconstruction technique. Eur Radiol 2012;22:1613-23

28. Yu L, Liu X, Leng S, et al. Radiation dose reduction in computed tomography: techniques and future perspective. Imaging Med 2009; 1:65-84

29. Szucs-Farkas Z, Bensler S, Torrente JC, et al. Nonlinear three-dimensional noise filter with low-dose CT angiography: effect on the detection of small high-contrast objects in a phantom model. Radiology 2011;258:261-69 